\title{
Anxiety and Cardiovascular Symptoms: The Modulating Role of Insomnia
}

\author{
O. Olafiranye ${ }^{a, d}$ G. Jean-Louis ${ }^{a, b, e}$ C. Magai ${ }^{f}$ F.Zizi ${ }^{a, b, e} \quad$ C.D. Brown ${ }^{a}$ \\ M. Dweck ${ }^{c}$ J.S. Borer ${ }^{d}$ \\ a Brooklyn Health Disparities Center, Department of Medicine, b Sleep Disorders Center, Department of Neurology, \\ Departments of ${ }^{\mathrm{C} O p h t h a l m o l o g y}$ and ${ }^{\mathrm{d}}$ Medicine, SUNY Downstate Medical Center, ${ }^{\mathrm{e} B r o o k l y n}$ Research

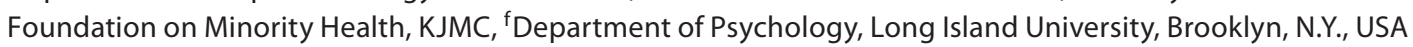

\section{Key Words}

Anxiety $\cdot$ Insomnia $\cdot$ Cardiovascular symptoms

\begin{abstract}
Background: Anxiety and insomnia are associated with cardiovascular (CV) symptoms. We assessed whether the relation between anxiety and CV symptoms is modulated by insomnia. Methods: Independently living women $(n=1,440$; mean age $=59.36 \pm 6.53$ years) were recruited by cluster sampling technique. We obtained data on demographic characteristics, health beliefs, access to health care, CV symptoms, sleep, stress and anxiety levels. Results: Overall, 56\% of the sample reported insomnia; $46 \%$ reported CV symptoms, and $54 \%$ were highly anxious. There was a greater likelihood for highly anxious women and those experiencing insomnia to report CV symptoms $\left(r_{s}=0.31^{*}\right.$ and $r_{s}=0.32^{*}$, respectively). In logistic regression analysis, the adjusted odds ratios for reporting CV symptoms were 1.39 for patients with insomnia and 2.79 for those with anxiety. With control for insomnia, we observed a 3-fold reduction in the magnitude of the association between anxiety and CV symptoms $\left(r_{p}=0.09 *\right)$. Stepwise adjustments for sociodemographic factors, CV risk markers, and factors anchoring health beliefs and access to health care showed lesser impact on the relationships. With simultaneous control for those covariates,
\end{abstract}

the correlation was $r_{p}=0.13^{*} ; p<0.01$. Conclusion: The association of CV symptoms with anxiety is partly accounted for by insomnia.

Copyright $\odot 2009$ S. Karger AG, Basel

\section{Introduction}

Anxiety and insomnia are both associated with cardiovascular (CV) disease. Evidence suggests that intensity of anxiety is directly associated with clinical manifestations of coronary heart disease and with sudden cardiac death [1-3]. Although the exact mechanism is unknown, a number of deleterious health behaviors (sedentary lifestyle, smoking, and poor diet) may contribute to the experience of negative emotions, such as anxiety [3]. Anxiety is associated with abnormal plasma norepinephrine and reactive oxygen species [4], which may contribute to atherosclerosis $[5,6]$. In two prospective cohort studies involving men, high levels of anxiety were associated with risk of coronary heart disease death $[7,8]$. Among women without $\mathrm{CV}$ disease or cancer, high levels of anxiety were associated with risk of fatal coronary events [9]. However, in these studies, statistical analysis indicated that anxiety did not account for the entirety of risk. Therefore, other factors need to be identified to ful- 
Table 1. Demographic characteristics of participating women based on ethnicity

\begin{tabular}{|c|c|c|c|c|c|c|c|c|}
\hline Variable & $\begin{array}{l}\text { AA } \\
(n=303)\end{array}$ & $\begin{array}{l}\text { EC } \\
(n=304)\end{array}$ & $\begin{array}{l}\mathrm{H} \\
(\mathrm{n}=309)\end{array}$ & $\begin{array}{l}\mathrm{D} \\
(\mathrm{n}=165)\end{array}$ & $\begin{array}{l}\mathrm{EE} \\
(\mathrm{n}=156)\end{array}$ & $\begin{array}{l}\text { EA } \\
(n=157)\end{array}$ & $\mathrm{F}$ & Post-hoc comparisons \\
\hline Mean age, years & $58.9 \pm 6.2$ & $58.4 \pm 7.0$ & $60.4 \pm 6.5$ & $58.3 \pm 6.1$ & $60.9 \pm 6.1$ & $59.6 \pm 6.5$ & $6.1^{*}$ & $\mathrm{H}, \mathrm{EE}>\mathrm{AA}, \mathrm{EC}$, and $\mathrm{D}$ \\
\hline Mean income, USD & $30.0 \pm 26.5$ & $34.9 \pm 20.3$ & $22.5 \pm 15.5$ & $25.3 \pm 14.5$ & $30.1 \pm 22.7$ & $46.3 \pm 34.6$ & $26.5^{* *}$ & $\mathrm{EA}>$ all; $\mathrm{EC}>\mathrm{H}, \mathrm{D} ; \mathrm{EE}>\mathrm{H}$ \\
\hline Mean education, years & $13.5 \pm 12.1$ & $13.3 \pm 8.3$ & $7.4 \pm 8.1$ & $7.2 \pm 8.2$ & $16.2 \pm 13.0$ & $14.3 \pm 13.1$ & $194.4^{* *}$ & $\mathrm{EE}>$ all, EA, AA, and EC $>\mathrm{H}$ and $\mathrm{D}$ \\
\hline
\end{tabular}

Values for mean income indicate thousands. Analysis of variance was used to assess ethnic differences in demographic factors.

$\mathrm{AA}=$ US-born African-American; EC = English-speaking Caribbean; H = Haitian; D = Dominican; EE = Eastern European; EA = US-born European American. ${ }^{*} \mathrm{p}<0.05,{ }^{* *} \mathrm{p}<0.01$.

ly understand the basis of coronary risk in anxious patients [9].

Evidence from several prospective studies suggests that insomnia may account for some of the risk not accounted for by anxiety in the association of anxiety with CV symptoms [10-13]. In addition, pathophysiological processes potentially important in generating $\mathrm{CV}$ events have been associated with insomnia [14]. The interrelation of anxiety, insomnia and CV symptoms has not been rigorously assessed. Therefore, in a prospective cohort study, we tested the hypothesis that the relation of anxiety and $\mathrm{CV}$ symptoms is significantly modulated by the presence or absence of insomnia.

\section{Methods}

\section{Participants}

The study population comprised 1,440 women residing in Brooklyn, N.Y. Self-ascribed ethnic identity was as follows: English-Speaking Caribbeans (22\%), Haitians (22\%), US-born BlackAfrican Americans (22\%), Dominicans (12\%), US-born WhitesEuropean Americans (11\%) and Eastern Europeans (11\%). Demographic characteristics of the sample are reported in table 1. Thirty-six women who did not fall into any of the designated ethnic groupings were excluded.

\section{Procedures}

Participants were recruited using a stratified, cluster sampling technique. Initially, data on census blocks were gathered from the Household Income and Race Summary Tape file 3A of the Census files. Blocks were then stratified by ethnic group (Whites, Blacks and Hispanics) and by income (low, medium, and high). Random selection without replacement was used to choose samples of block groups from each stratum, and only women who were 50 years old or older were recruited.

During face-to-face interviews lasting approximately $11 / 2 \mathrm{~h}$, prespecified data were collected by trained interviewers of the same ethnicity as the participants. Interviews were conducted either in the respondent's home or at another location of the respondent's choice. Participants received USD 25 upon completion of the interview. In a standard order, several scales/questionnaires were administered to elicit subjective and demographic data. Physical health was measured with the Comprehensive Assessment and Referral Evaluation (CARE) $[15,16]$. Measures for the present analysis included demographic, health, risk markers, and sleep complaints.

The CARE is regularly used to assess physical health, particularly among individuals in minority communities. Psychometric studies have shown that it has good construct validity $[15,16]$ as well as concurrent and predictive validity [15]; subscales considered in our analyses were somatic, sleep disorder, leg problem, heart disease, respiratory disease, arthritis, vision problem, and hypertension (Cronbach's $\alpha=0.86 ; 0.85 ; 0.86 ; 0.83 ; 0.72 ; 0.91$; 0.85 , and 0.92 , respectively). Items from the subscales were not aggregated, as the analysis focused only on the presence or absence of symptoms of medical conditions. Thus, rates of medical problems may not of necessity reflect physician-diagnosed illnesses.

The sleep disorder subscale includes five questions: Do you depend on medicine to sleep? Do you have difficulty falling asleep? Do you wake up often during the night? Do you wake up early and wake up feeling tired? Do you sleep during the day? The present analysis considers the three most commonly reported insomnia symptoms, namely difficulty initiating sleep, difficulty maintaining sleep, and early morning awakening. Thus, the insomnia symptom variable represents an aggregate of these three complaints. Women were categorized into two groups, those who experienced any of the symptoms vs. those who did not. No formal insomnia diagnosis could be formulated, as data on frequency, duration, or severity were not elicited. The variables daytime sleep (nap) and use of sleep medicine were used only in comparative analyses. They were not considered in the final statistical model, as they tend to show relatively poor association with insomnia.

The CV subscale includes several questions assessing the presence of CV symptoms. CV questions were as follows: Have you ever had palpitations due to a medical condition? Do you experience breathlessness lying flat, which is relieved when standing? Have you ever had heart trouble? Do you have heart problem that was diagnosed by a doctor? Have you experienced heart problems in the last month? Do you experience chest pain on exertion or made worse by exertion? Have you experienced chest pain that is relieved by rest or nitroglycerine? Have you experienced uncomfortable feeling of chest pain behind lower $1 / 2$ of the sternum? Have you experienced severe chest pain after age 40 ? Have you experienced chest pain in last year but not last month? 
Table 2. Comparison of risk markers of participants based on ethnicity

\begin{tabular}{|c|c|c|c|c|c|c|c|c|}
\hline Variable & $\mathrm{AA}$ & EC & $\mathrm{H}$ & $\mathrm{D}$ & $\mathrm{EE}$ & EA & $\mathrm{F} / \chi^{2}$ & Post-hoc comparisons \\
\hline BMI & $30.9 \pm 6.6$ & $29.7 \pm 4.5$ & $28.8 \pm 5.0$ & $28.5 \pm 5.0$ & $29.2 \pm 6.3$ & $27.5 \pm 5.7$ & $10.8^{* *}$ & $\mathrm{AA}>\mathrm{H}, \mathrm{D}, \mathrm{EE}$, and $\mathrm{EA} ; \mathrm{EC}>\mathrm{EA}$ \\
\hline Stress (0-29) & $8.6 \pm 6.2$ & $10.9 \pm 6.4$ & $8.5 \pm 6.9$ & $11.7 \pm 5.4$ & $10.9 \pm 6.0$ & $8.8 \pm 5.1$ & $12.3^{* *}$ & $\begin{array}{l}\mathrm{AA}<\mathrm{EC}, \mathrm{D}, \text { and EE; EC }>\text { EA; } \\
\mathrm{H}>\mathrm{D} \text { and EE; D }>\mathrm{EA} ; \mathrm{EE}>\mathrm{EA}\end{array}$ \\
\hline Smoking, \% & 27 & 5 & 2 & 10 & 8 & 12 & $117^{* *}$ & $\begin{array}{l}\mathrm{AA}>\mathrm{ALL} ; \mathrm{D}>\mathrm{EC} \text { and } \mathrm{H} ; \\
\mathrm{EA}>\mathrm{EC}, \mathrm{H}, \text { and } \mathrm{EE} ; \mathrm{EE}>\mathrm{H}\end{array}$ \\
\hline Drinking, \% & 36 & 15 & 4 & 39 & 24 & 46 & $166^{* *}$ & $\begin{array}{l}\mathrm{EA}>\mathrm{ALL} ; \mathrm{AA} \text { and } \mathrm{D}>\mathrm{EC}, \mathrm{H}, \\
\text { and EE; EC }>\mathrm{H}\end{array}$ \\
\hline
\end{tabular}

Ethnic differences in risk markers for insomnia complaints and anxiety were assessed with ANOVA/Fisher's exact tests.

** $\mathrm{p}<0.01$.

Table 3. Comparison of health characteristics of participants based on ethnicity

\begin{tabular}{lllllllll}
\hline Variable & AA & EC & H & D & EE & EA & $\chi^{2}$ & Comparison \\
\hline Respiratory disease, \% & 55 & 16 & 15 & 61 & 47 & 59 & $243^{* *}$ & $\begin{array}{l}\text { AA, D and EA }>\text { EC and H; EE }>\text { EC and H; } \\
\text { EA and D }>\text { EE }\end{array}$ \\
\hline Hypertension, \% & 55 & 61 & 56 & 54 & 51 & 34 & $34^{* *}$ & EA $<$ all \\
\hline Heart disease, \% & 48 & 20 & 54 & 58 & 75 & 36 & $162^{* *}$ & EE $>$ all; AA, H and D $>$ EC and EA; EA $>$ EC \\
\hline Arthritis, \% & 73 & 51 & 77 & 80 & 79 & 67 & $73^{* *}$ & EC $<$ ALL; EA $<$ D and EE \\
\hline
\end{tabular}

Ethnic differences in health characteristics were assessed using Fisher's exact tests. ${ }^{* *} \mathrm{p}<0.01$.

Have you experienced chest pain in last month? Have you experienced chest pain that is not relieved by rest? Have you ever had a stroke or sudden paralysis or loss of speech? Do you have paralysis or weakness in one arm or leg? In preliminary analyses, responses to $\mathrm{CV}$ questions were associated with the insomnia variable $(\mathrm{p}<0.05)$.

The stress index scale used by the National Survey of Black Americans was administered to our participants $[15,17]$. Respondents rated on a 4-point scale the degree to which a set of items provoked stress in the past 2 months. These stress-induced life events were health, money, job, problems with family or marriage, problems with people outside the family, children, crime, police, love life, and racial conflict. Scores ranged from 0 to 29; higher scores indicated greater stress levels $(\alpha=0.81)$. We used the StateTrait Anxiety Inventory to assess anxiety level $(\alpha=0.75)$ [17]. It assesses how respondents felt at a particular time in the recent past and how they anticipated they would feel either in a specific situation that is likely to be encountered in the future or in a variety of hypothetical situations. Scores in that scale ranged from 0 to 24 ; higher scores reflect greater state anxiety.

\section{Statistical Analysis}

For sample description, frequency and measures of central tendency were used. Variables were examined for normality and tested for collinearity. Skewed distributions were transformed us- ing appropriate statistical techniques. Analysis of variance was used to assess possible ethnic differences in continuous variables. Fisher's exact test was employed to assess ethnic differences in demographic factors, risk markers, and physical health characteristics. As baseline measures were influenced by women's ethnicity, the ethnic variable was treated as a covariate in the final regression model.

We used logistic regression analysis to examine which factors were predictive of the dependent variable, CV symptoms. The dependent variable was a binary measure, classifying women into two groups, those reporting CV symptoms vs. those reporting no $\mathrm{CV}$ symptoms. In the present analyses, the initial predictors were the insomnia and anxiety factors. Three other sets of factors were entered in the model in a stepwise manner. They included: sociodemographics (i.e. ethnicity, age, education, and income), risk markers (i.e. BMI, smoking, drinking, and stress), and medical factors (i.e. respiratory disease, arthritis, and hypertension).

\section{Results}

Risk markers and health characteristics of participating women are provided in tables 2 and 3. Overall, 56\% of the sample reported insomnia (defined as difficulty 


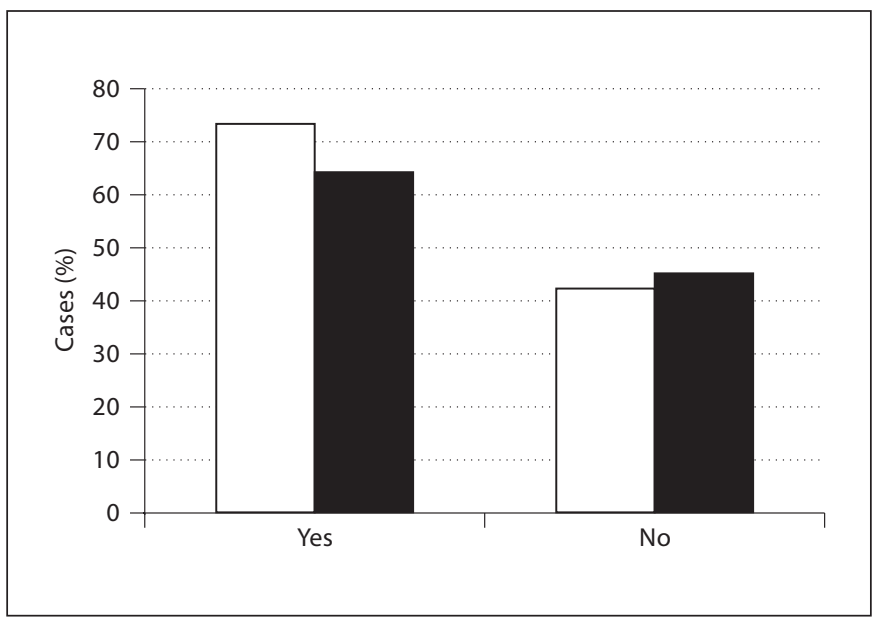

Fig. 1. Illustration of differences in the rate of insomnia ( $\square$ ) and anxiety ( $\square$ ) comparing women reporting having experienced CV symptoms (yes) vs. those who did not (no). Comparisons were significant using Fisher's exact test at $\alpha=0.01$.

initiating sleep, difficulty maintaining sleep, or early morning awakening). CV symptoms were reported in $46 \%$; $54 \%$ were classified as highly anxious. As expected, there was a greater likelihood for highly anxious women and those experiencing insomnia to report CV symptoms $\left(\mathrm{r}_{\mathrm{s}}=0.31, \mathrm{p}<0.01\right.$ and $\mathrm{r}_{\mathrm{s}}=0.32, \mathrm{p}<0.01$, respectively). Of the women reporting CV symptoms, $63 \%$ were highly anxious $\left(\chi^{2}=44.86, \mathrm{p}<0.01\right)$ and $73 \%$ experienced insomnia $\left(\chi^{2}=139.06, \mathrm{p}<0.01\right.$; fig. 1$)$. Of interest is the observation that all of the $\mathrm{CV}$ symptoms were associated with insomnia $(\mathrm{p}<0.05)$. Notably, $5 \%$ of individuals reporting insomnia symptoms were likely to indicate having had a stroke or sudden paralysis or loss of speech, compared with $2 \%$ of those who did not report insomnia $\left(\chi^{2}=8.46, \mathrm{p}<0.01\right)$.

Results of logistic regression analysis revealed adjusted odds ratios of 1.39 (insomnia) and 2.79 (anxiety) for reporting CV symptoms. With statistical control for insomnia, we observed a 3 -fold reduction in the magnitude of the associations between anxiety and CV symptoms $\left(r_{p}=0.09, p<0.05\right)$. Stepwise adjustments for sociodemographic factors (i.e. age, ethnicity, education, and income), risk makers (i.e. BMI, stress, smoking, and alcohol), and medical factors showed lesser impact on the relationships $\left(\mathrm{r}_{\mathrm{p}}=0.14, \mathrm{p}<0.05 ; \mathrm{r}_{\mathrm{p}}=0.16, \mathrm{p}<0.05 ; \mathrm{r}_{\mathrm{p}}=\right.$ $0.19, \mathrm{p}<0.05$, respectively). With simultaneous control for those covariates, the correlation was $r_{p}=0.13, p<$ 0.05 .

Anxiety and Cardiovascular Symptoms

\section{Discussion}

The present study showed that the association of CV symptoms with anxiety is in part accounted for by insomnia. Adjustment for potential confounding effects of sociodemographic factors and CV risk markers had little impact on the observed relationships. Converging data indicate that anxiety and insomnia often coexist $[18,19]$. Since these factors cluster together in variable fashion, it is difficult to discern the potential mechanism by which they exert their pathophysiological effects. Autonomic dysfunction, reduced heart rate variability [20], and reduced baroreflex cardiac control observed among patients with anxiety disorders [21] have been suggested as the pathophysiological link between anxiety and CV symptoms. Since anxiety could not account for all of the risks noted in previous research, other factors might play a contributory role [9].

Our finding of 3-fold reduction in the association between anxiety and CV symptoms after controlling for insomnia suggests that insomnia plays a vital role in the development of CV symptoms among women with anxiety. Despite growing evidence supporting adverse effects of insomnia on CV health, the pathogenesis of this relation is not completely understood. Since insomnia is a sympathetic disorder, it might place susceptible individuals at increased cardiac risk. One possible explanation relates to the impairment in the normal restoration process occurring during slow-wave sleep [13] which few patients with insomnia experience. This is consistent with a previous report suggesting that insomnia could be a marker for chronic stress, which is accompanied by autonomic dysfunction and increased myocardial infarction [22]. Sleep disturbance in and of itself can constitute a form of stress [14], which is directly related to the degree of objective sleep disturbance [23] and has been shown to predict coronary events, possibly mediated through increased sympathetic nervous system activity [1]. A study has also shown that individuals with chronic insomnia suffer from increased arousal and sympathetic activation [24]. This could be the result of nonrestorative sleep function, immune dysfunction and carbohydrate intolerance secondary to sleep loss [25-27].

Investigators have observed that sympathetic arousal and early-morning insomnia were associated with cardiac disease in a sample of 4,041 patients [10]. Among older adults, insomnia severity was directly related to use of CV drugs, general fatigue, exhaustion, angina pectoris, cardiac insufficiency, worsened objective and subjective health, presence of negative T-waves on ECG and lower

Cardiology 2010;115:114-119 
survival rates [11]. In another study, investigators observed that a combination of three sleep complaints (difficulty initiating sleep, difficulty maintaining sleep, and awakening tired and fatigued) predicted risk of CV disease [12]. In agreement with a prior study [28], we observed an increased risk of stroke in women reporting insomnia.

The high percentage of women reporting anxiety in association with insomnia in the present study is consistent with published data $[21,29]$. Disturbed sleep is a diagnostic criterion in generalized anxiety disorder. In a study involving 176 undergraduate students, social anxiety was associated with insomnia symptoms [30]. Another study sampling 424 noninstitutionalized elderly patients showed that anxiety disorders were associated with primary insomnia [31]. In 3 large-scale community-based studies, including one involving 34,000 men, significant relationships between anxiety disorders and cardiac death were observed $[2,7,8]$. However, these studies did not include women. A particular strength of the present study relates to the fact that that we focused on women, a group often characterized by higher prevalence of anxiety disorder [18] and sleep problems than men [25, 26,32]. This is important since high levels of anxiety among women have been associated with risk of fatal coronary heart disease [9]. Another streng th of this study is the fact that the majority of our study population are Caribbean, Haitian and African-American women. These unique ethnic groups are of special interest since Blacks are in general understudied and underserved, despite being at greater risk for $\mathrm{CV}$ events.

This study has several limitations. First, our findings may not be generalized to men and younger females as only women older than 50 years were enrolled. Other studies have shown clear evidence of neurobiological gender differences in sleep regulation [33]. Second, we did not collect objective data on sleep time and patterns to examine the severity of sleep complaints. However, the definition of insomnia is dependent on the subjective experience of disturbed sleep [34]. Sleep complaint is also important, as it anchors a person's likelihood of seeking medical advice or treatment. Furthermore, self-reports of sleep are the measures most widely used in communitybased epidemiological surveys, and there is evidence that subjective evaluations correlate reasonably with objective laboratory data [35]. Our analysis was based on subjective report of CV symptoms, with no verifiable clinical diagnosis by a physician. Future studies should include men and younger women and also examine these associations using objective data. It is of particular interest to examine whether individuals reporting insomnia symptoms would be more likely to have experienced a stroke, as observed from our subjective data.

In conclusion, this study showed that the association of CV symptoms with anxiety is in part accounted for by insomnia. If this finding is confirmed in other studies, it may be useful to assess the impact of treatment of insomnia on CV symptoms and events.

\section{Acknowledgement}

This research was supported by NIH (SO6 GM54650, R24MD001090, R01MD004113, P20MD005092, and HL085042).

\section{References}

1 Rozanski A, Blumenthal JA, Kaplan J: Impact of psychological factors on the pathogenesis of cardiovascular disease and implications for therapy. Circulation 1999;99: 2192-2217.

-2 Kawachi I, Sparrow D, Vokonas PS, Weiss ST: Symptoms of anxiety and risk of coronary heart disease. The Normative Aging Study. Circulation 1994;90:2225-2229.

3 Kubzansky LD, Kawachi I: Going to the heart of the matter: negative emotions and coronary heart disease. J Psychosom Res 2000;48:323-337.

4 Yasunari K, Matsui T, Maeda K, Nakamura M, Watanabe T, Kiriike N: Anxiety-induced plasma norepinephrine augmentation increases reactive oxygen species formation by monocytes in essential hypertension. Am J Hypertens 2006;19:573-578.
5 Ross R: Atherosclerosis - an inflammatory disease. N Eng J Med 1999;340:115-126.

6 Alexander RW: Theodore Cooper Memorial Lecture. Hypertension and the pathogenesis of atherosclerosis. Oxidative stress and the mediation of arterial inflammatory response: a new prospective. Hypertension 1995;25:155-161.

$\checkmark$ Haines AP, Imeson JD, Meade TW: Phobic anxiety and ischemic heart disease. BMJ 1987;295:297-299.

-8 Kawachi I, Colditz GA, Ascherio A, Rimm EB, Giovannucci E, Stampfer MJ, Willet WC: Coronary heart disease/myocardial infarction: prospective study of phobic anxiety and risk of coronary heart disease in men. Circulation 1994;89:1992-1997.
9 Albert CM, Chae CU, Rexrode KM, Manson JE, Kawachi I: Phobic anxiety and risk of coronary heart disease and sudden cardiac death among women. Circulation 2005;111: 480-487.

10 Fraguas RJ, Iosifescu DV, Alpert J, Wisniewski SR, Barkin JL, Trivedi MH, Rush AJ, Fava M: Major depressive disorder and comorbid cardiac disease: is there a depressive subtype with greater cardiovascular morbidity? Results from the STAR*D study. Psychosomatics 2007;48:418-425.

11 Jensen E, Dehlin O, Hagberg B, Samuelsson $\mathrm{G}$, Svensson T: Insomnia in an 80-year-oldpopulation: relationship to medical, psychological and social factors. J Sleep Res 1998;7: 183-189. 
12 Phillips B, Mannino DM: Do insomnia complaints cause hypertension or cardiovascular disease. J Clin Sleep Med 2007;3:489-494.

13 Van Diest R, Appels WP: Sleep physiological characteristics of exhausted men. Psychosom Med 1994;56:28-35.

14 Terzano MG, Parrino L, Mennuni GF: Phasic events and microstructure of sleep. Consensus Conference, Martano, Italian Association of Sleep Medicine (AIMS), Lecce, 1997.

-15 Teresi JA, Golden RR, Gurland BJ, Wilder DE, Bennett RG: Construct validity of indicator-scales developed from the Comprehensive Assessment and Referral Evaluation interview schedule. J Gerontol 1984;39:147157.

- 16 Teresi JA, Golden RR, Gurland BJ: Concurrent and predictive validity of indicator scales developed for the Comprehensive Assessment and Referral Evaluation interview schedule. J Gerontol 1984;39:158-165.

-17 Bieling PJ, Antony MM, Swinson RP: The State-Trait Anxiety Inventory, Trait version: structure and content re-examined. Behav Res Ther 1998;36:777-788.

-18 Shehan DV: Current concepts in psychiatry: panic attacks and phobias. N Engl J Med 1982;307:156-158.

$\checkmark 19$ Anderson DJ, Noyes R, Crowe RR: A comparison of panic disorder and generalised anxiety disorder. Am J Psychiatry 1984;141: 572-575.
20 Kawachi I, Sparrow D, Vokonas PS, Weiss ST: Decreased heart rate variability in men with phobic anxiety. Data from the Normative Aging Study. Am J Cardiol 1995;75:882885.

21 Watkins LL, Grossman P, Krishnan R, Sherwood A: Anxiety and vagal control of heart risk. Psychosom Med 1998;60:498-502.

22 Schwartz SW, Cornoni-Huntley J, Cole SR, Hays JC, Blazer DG, Schocken DD: Are sleep complaints an independent risk factor for myocardial infarction? Ann Epidemiol 1998; 8:384-392.

23 Vgontzas AN, Tsigos C, Bixler EO, et al Chronic insomnia and activity of the stress system: a preliminary study. J Psychosom Res 1998;45:21-31.

24 Bonnet M, Arand D: Activity, arousal and the MSLT in patients with insomnia. Sleep 2000;23:205-212.

25 Mallon L, Broman LG, Hetta J: Sleep complaints predict coronary artery disease mortality in males: a 12-year follow-up study of a middle-aged Swedish population. J Int Med 2002;251:207-216.

-26 Nilsson PM, Nilsson JA, Hedblad B, Berglund G: Sleep disturbance in association with elevated pulse rate for prediction of mortality-consequences of mental strain? J Int Med 2001;250:521-529.

27 Vgontzas AN, Chrousos GP: Sleep, the hypothalamic-pituitary-adrenal axis, and cytokines multiple interactions and disturbances in sleep disorders. Endocrinol Metab Clin North Am 2002;31:15-36.

28 Chen JC, Brunner RL, Ren H, WassertheilSmoller S, Larson JC, Levine DW, Allison M, Naughton MJ, Stefanick ML: Sleep duration and risk of ischemic stroke in postmenopausal women. Stroke 2008;39:3185-3192.
29 Hoehn-Saric R: Characteristics of chronic anxiety patients; in Klein DF, Rabkin JG (eds): Anxiety: New Research and Changing Concepts. New York, Raven Press, 1981.

>30 Buckner JD, Bernert RA, Cromer KR, Joiner TE, Schmidt NB: Social anxiety and insomnia: the mediating role of depression. Depress Anxiety 2008;25:124-130.

31 Hidalgo JL, Gras CB, Garcia YD, Lapeira JT, del Campo JM, Verdejo MA: Functional status in the elderly with insomnia. Qual Life Res 2007;16:279-286.

-32 Billing E, Hjemdahl P, Rehnqvist N: Psychosocial variables in female vs male patients with stable angina pectoris and matched healthy controls. Euro Heart J 1997;18:911918.

33 Armitage R, Hoffmann RF: Sleep EEG, depression and gender. Sleep Med Rev 2001;5: 237-246.

34 Diagnostic Classification Steering Committee, Thorpy MJ, Chairman: ICSD - International Classification of Sleep Disorders; Diagnostic and Coding Manual. Rochester, American Sleep Disorders Association, 1990.

35 Vanable P, Aikens J, Tadimeti L, CaruanaMontaldo B, Mendelson W: Sleep latency and duration estimates among sleep disorder patients: variability as a function of sleep disorder diagnosis, sleep history, and psychological characteristics. Sleep 2000;23:71-79. 